\title{
Prime tight frames
}

\section{Lemvig, Jakob; Miller, Christopher; Okoudjou, Kasso A.}

\section{Published in:}

Advances in Computational Mathematics

Link to article, DOI:

10.1007/s10444-013-9309-0

Publication date:

2014

\section{Document Version}

Early version, also known as pre-print

Link back to DTU Orbit

Citation (APA):

Lemvig, J., Miller, C., \& Okoudjou, K. A. (2014). Prime tight frames. Advances in Computational Mathematics, 40(2), 315-334. https://doi.org/10.1007/s10444-013-9309-0

\section{General rights}

Copyright and moral rights for the publications made accessible in the public portal are retained by the authors and/or other copyright owners and it is a condition of accessing publications that users recognise and abide by the legal requirements associated with these rights.

- Users may download and print one copy of any publication from the public portal for the purpose of private study or research.

- You may not further distribute the material or use it for any profit-making activity or commercial gain

- You may freely distribute the URL identifying the publication in the public portal

If you believe that this document breaches copyright please contact us providing details, and we will remove access to the work immediately and investigate your claim. 


\title{
Prime tight frames
}

\author{
Jakob Lemvig*, Christopher Miller, and Kasso A. Okoudjou* \\ July 31,2012
}

\begin{abstract}
We introduce a class of finite tight frames called prime tight frames and prove some of their elementary properties. In particular, we show that any finite tight frame can be written as a union of prime tight frames. We then characterize all prime harmonic tight frames and use this characterization to suggest effective analysis and synthesis computation strategies for such frames. Finally, we describe all prime frames constructed from the spectral tetris method, and, as a byproduct, we obtain a characterization of when the spectral tetris construction works for redundancies below two.
\end{abstract}

\section{Introduction}

A frame for a finite dimensional vector space is a spanning set that is not necessarily a basis. More specifically, let $\mathbb{K}$ denote either $\mathbb{R}$ or $\mathbb{C}$, and consider $\mathbb{K}^{N}, N \geq 1$, as a vector space over the scalar field $\mathbb{K}$. Given $M \geq N$, a collection of vectors $\Phi=\left\{\varphi_{i}\right\}_{i=1}^{M} \subset \mathbb{K}^{N}$ is called a finite frame for $\mathbb{K}^{N}$ if there are two constants $0<A \leq B$ such that

$$
A\|x\|^{2} \leq \sum_{i=1}^{M}\left|\left\langle x, \varphi_{i}\right\rangle\right|^{2} \leq B\|x\|^{2}, \quad \text { for all } x \in \mathbb{K}^{N} .
$$

If the frame bounds $A$ and $B$ are equal, the frame $\Phi=\left\{\varphi_{i}\right\}_{i=1}^{M}$ is called a finite tight frame. We refer to $\Phi=\left\{\varphi_{i}\right\}_{i=1}^{M}$ as a finite unit norm tight frame (FUNTF), if $\Phi$ is a tight frame with $\left\|\varphi_{i}\right\|=1$ for each $i$. In this case, the frame bound is $A=M / N$. For a tight frame $\Phi=\left\{\varphi_{i}\right\}_{i=1}^{M}$ with frame bound $A$, the following reproducing formula holds

$$
x=\frac{1}{A} \sum_{i=1}^{M}\left\langle x, \varphi_{i}\right\rangle \varphi_{i}, \quad \text { for all } x \in \mathbb{K}^{N} .
$$

*Technical University of Denmark, Department of Mathematics, Matematiktorvet 303, 2800 Kgs. Lyngby, Denmark, E-mail: J.Lemvig@mat.dtu.dk

$\dagger 8803$ Pisgah Drive Clinton MD, 20735 USA, E-mail: christopherdmiller5@gmail.com

Department of Mathematics, University of Maryland, College Park, MD, 20742 USA, E-mail: kasso@math.umd.edu

2010 Mathematics Subject Classification. Primary 42C15; Secondary 11A07

Key words and phrases. divisible frames, equiangular tight frames, frames, harmonic tight frames, prime frames, spectral tetris frames, tight frames 
The decomposition formula provided by frames has important consequences in many areas of science and engineering in which they now play an increasingly important role. We refer to $[9,11,12]$ for an overview of frames and some of their applications. In particular, tight frames and FUNTFs have attracted a lot of attention in recent years due to their numerous applications. In this context methods for characterizing and constructing these types of frames are being actively investigated. For instance, the existence and characterization of FUNTFs was settled by Benedetto and Fickus in [2] as minimizers of a potential function.

In this paper we are interested in the classification of the tight frames that remain tight after the deletion of frame vectors. For (non-tight) frames, this problem is known as the erasure problem for frames and was first investigated by Goyal, Kovačević, and Kelner [10], and later by Casazza and Kovačević [7]. The focus in these works was whether any set of vectors of a given size can be removed from a frame to still leave a frame. In this case, estimates for frame bounds after erasures were given.

By contrast, we investigate tight frames which remain such after the erasures of some set of frame vectors. In the process, we define a new class of tight frames called prime tight frames as tight frames for which no proper sub-collection is a tight frame. In Section 2, we analyze the structure of prime tight frames. In particular, we show that prime tight frames are fundamental building blocks for all tight frames in the sense that any tight frame can be written as a union of prime tight frames. This is, in a way, analog to the prime factorization of natural numbers; however, this factorization of tight frames into prime ones is not unique. In Section 3, we then restrict our attention to FUNTFs and characterize the prime tight frames for three families of FUNTFs: equiangular tight frames, harmonic tight frames, and spectral tetris frames. Equiangular and harmonic tight frames have proven to be some of the most useful frames in a variety of applications. For harmonic tight frames (HTF), i.e., tight frames constructed from an $M \times M$ discrete Fourier matrix by keeping the first $N$ rows, this characterization leads to effective analysis and synthesis computation strategies for HTFs. The spectral tetris method was recently introduced by Casazza, Fickus, Mixon, Wang, and Zhou [5] and has already received considerable attention in the frame theory community. As a byproduct of our results on prime spectral tetris frames, we are able to characterize all dimensions $N$ and number of frame vectors $M$ for which the spectral tetris construction works. This was previously only known for redundancies larger than two.

\section{Basic facts of prime and divisible tight frames}

We define prime and divisible tight frames and prove some of their properties in Section 2.1. In Section 2.2, we then prove that prime frames exist in every dimension which allows us to conclude that prime tight frames form an open, dense subset of the set of all tight frames.

\subsection{Definitions and elementary properties}

Definition 2.1. Let $M \geq N$ be given. A tight frame $\Phi=\left\{\varphi_{i}\right\}_{i=1}^{M}$ in $\mathbb{K}^{N}$ is said to be prime, if no proper subset of $\Phi$ is a tight frame for $\mathbb{K}^{N}$. If $\Phi$ is not prime, we say that it is divisible. In particular, given an integer $p$ with $N \leq p \leq M-N$, the tight frame $\Phi$ is $(M, p)$-divisible if there exists a subset of $\Phi$ containing $p$ vectors that is also a tight frame.

Remark 1. For a tight frame $\Phi=\left\{\varphi_{i}\right\}_{i=1}^{M}$ in $\mathbb{K}^{N}$ to be prime it is sufficient that $M<2 N$. In other words, for $\Phi$ to be divisible it is necessary that $M \geq 2 N$. This follows from the fact that, 
if $M<2 N$, it is impossible to partition $\Phi$ into two spanning sets.

We also note that there exist frames $\Phi$ that are $(M, p)$-divisible for all $p$ in the full range $N \leq p \leq M-N$, e.g., choosing $\Phi$ to be the harmonic tight frame in $\mathbb{C}^{2}$ with 24 frame vectors. On the other hand, it is not possible for a tight frame to be robust with respect to tightness for any $p$ erasures, where $p \in \mathbb{N}$ is fixed. Hence within the class of tight frames the erasure problem has no solution. This negative result and a simple symmetry observation are stated in the next proposition.

Proposition 2.2. Let $M \geq N \geq 2$ be given. Let $\Phi=\left\{\varphi_{i}\right\}_{i=1}^{M}$ be a tight frame in $\mathbb{K}^{N}$.

(i) $\Phi$ is $(M, p)$-divisible if and only if $\Phi$ is $(M, N-p)$-divisible.

(ii) Given $N \leq p \leq M-N$, there exists no $(M, p)$-divisible tight frame $\Phi$ in $\mathbb{K}^{N}$ for which every sub-collection of $\Phi$ consisting of $p$ vectors is a tight frame.

Proof. (i): The proof of this part is trivial and so we omit it.

(ii): First assume that $\Phi=\left\{\varphi_{k}\right\}_{k=1}^{M}$ is a FUNTF that is $(M, p)$-divisible and such that every sub-collection of $p$ vectors is again a FUNTF. Let $\Phi_{1}=\left\{\varphi_{i}\right\}_{i \in J}$, where $J=\{1, \ldots, p\}$, be the tight frame of the first $p$ vectors from $\Phi$. We replace the $\ell$ th frame vector in $\Phi_{1}$ by the $k$ th vector in $\Phi$, where $p+1 \leq k \leq M$, and denote this tight frame $\Phi_{2}:=\left\{\varphi_{i}\right\}_{i \in J \backslash\{\ell\}} \cup\left\{\varphi_{k}\right\}$. Then, given any $x \in \mathbb{K}^{N}$, we have

$$
\frac{p}{N}\|x\|^{2}=\sum_{i \in J}\left|\left\langle x, \varphi_{i}\right\rangle\right|^{2}=\sum_{i \in J \backslash\{\ell\}}\left|\left\langle x, \varphi_{i}\right\rangle\right|^{2}+\left|\left\langle x, \varphi_{k}\right\rangle\right|^{2} .
$$

This implies that $\left|\left\langle x, \varphi_{\ell}\right\rangle\right|=\left|\left\langle x, \varphi_{k}\right\rangle\right|$. Since $\ell$ and $k$ are arbitrary, we have that $\left|\left\langle x, \varphi_{\ell}\right\rangle\right|=\left|\left\langle x, \varphi_{k}\right\rangle\right|$ for each $x \in \mathbb{K}^{N}$, each $\ell=1,2, \ldots, p$, and each $p+1 \leq k \leq M$. In particular, we have that $\left|\left\langle\varphi_{k}, \varphi_{\ell}\right\rangle\right|=1$ for $1 \leq \ell \leq p$ and $p+1 \leq k \leq M$. Since the vectors $\varphi_{i}$ are unit-norm, for a fixed $k=k_{0} \in\{p+1, \ldots, M\}$, this implies that $\varphi_{\ell} \in \operatorname{span}\left(\varphi_{k_{0}}\right)$ for all $1 \leq \ell \leq p$. Hence, the span of $\Phi_{1}$ is one-dimensional which contradicts the assumption that $\Phi_{1}$ is a tight frame. We conclude that there exist no FUNTF that is $(M, p)$-divisible and for which all sub-collections of $p$ vectors are also FUNTF. This proof carries over also to the case of equal norm tight frames.

Now suppose that $\Phi=\left\{\varphi_{i}\right\}_{i=1}^{M}$ is an $(M, p)$-divisible non-equal norm, tight frame such that any subset of $p$ vectors is again a tight frame. Assume without loss of generality that $\left\|\varphi_{1}\right\| \geq\left\|\varphi_{2}\right\| \geq \ldots \geq\left\|\varphi_{M}\right\|$. Let $\Phi_{1}=\left\{\varphi_{i}\right\}_{i=1}^{p}$ be the subset of the first $p$ vectors from $\Phi$, and let $\Phi_{2}=\left\{\varphi_{i}\right\}_{i=1}^{p-1} \cup\left\{\varphi_{k}\right\}$ where $p+1 \leq k \leq M$, $p$ being the first index such that $\left\|\varphi_{p}\right\|>\left\|\varphi_{k}\right\|$. Then, given any $x \in \mathbb{K}^{N}$, we have

$$
\frac{\sum_{i=1}^{p}\left\|\varphi_{i}\right\|^{2}}{N}\|x\|^{2}=\sum_{i=1}^{p}\left|\left\langle x, \varphi_{i}\right\rangle\right|^{2} \quad \text { and } \quad \frac{\sum_{i=1}^{p-1}\left\|\varphi_{i}\right\|^{2}+\left\|\varphi_{k}\right\|^{2}}{N}\|x\|^{2}=\sum_{i=1}^{p-1}\left|\left\langle x, \varphi_{i}\right\rangle\right|^{2}+\left|\left\langle x, \varphi_{k}\right\rangle\right|^{2} .
$$

This implies that $\left|\left\langle x, \varphi_{p}\right\rangle\right|^{2}-\left|\left\langle x, \varphi_{k}\right\rangle\right|^{2}=\frac{\left\|\varphi_{p}\right\|^{2}-\left\|\varphi_{k}\right\|^{2}}{N}\|x\|^{2}$ for each $x \in \mathbb{K}^{N}$. If we choose $x=\varphi_{p}$, we find that

$$
\left\|\varphi_{p}\right\|^{4}-\left|\left\langle\varphi_{p}, \varphi_{k}\right\rangle\right|^{2}=\frac{\left\|\varphi_{p}\right\|^{2}-\left\|\varphi_{k}\right\|^{2}}{N}\left\|\varphi_{p}\right\|^{2},
$$

and similarly, for $x=\varphi_{k}$, we have

$$
\left|\left\langle\varphi_{p}, \varphi_{k}\right\rangle\right|^{2}-\left\|\varphi_{k}\right\|^{4}=\frac{\left\|\varphi_{p}\right\|^{2}-\left\|\varphi_{k}\right\|^{2}}{N}\left\|\varphi_{k}\right\|^{2} .
$$


Combining these two equations we obtain

$$
\left\|\varphi_{p}\right\|^{4}-\left\|\varphi_{k}\right\|^{4}=\frac{\left\|\varphi_{p}\right\|^{4}-\left\|\varphi_{k}\right\|^{4}}{N}
$$

which leads to $N=1$. This contradiction concludes the proof.

The following result shows that if we can take out a subset of vectors from a tight frame such that these vectors form a tight frame, then what is left is automatically also a tight frame.

Theorem 2.3. Let $M \geq 2 N$. Suppose $\Phi=\left\{\varphi_{k}\right\}_{k=1}^{M}$ is a divisible tight frame for $\mathbb{K}^{N}$, and let $\Phi_{1} \subsetneq \Phi$ denote a tight frame for $\mathbb{K}^{N}$. Then $\Phi_{2}=\Phi \backslash \Phi_{1}$ is also a tight frame for $\mathbb{K}^{N}$.

Moreover, given $M \geq N$, every tight frame of $M$ vectors in $\mathbb{K}^{N}$ is a finite union of prime tight frames.

Proof. Assume that $\Phi$ and $\Phi_{1} \subsetneq \Phi$ are tight frames with frame bounds $A$ and $A_{1}$, respectively, and let $\Phi_{2}=\Phi \backslash \Phi_{1}$. We will now consider $\Phi$ as an $N \times M$ matrix of the form $\Phi=\left[\varphi_{i}\right]_{i=1}^{M} \in$ $\operatorname{Mat}(N \times M, \mathbb{K})$. Hence, after a possible reordering of columns, we have that $\Phi=\left[\Phi_{1} \Phi_{2}\right]$, and

$$
A I_{N}=\Phi \Phi^{*}=\left[\Phi_{1} \Phi_{2}\right]\left[\begin{array}{l}
\Phi_{1}^{*} \\
\Phi_{2}^{*}
\end{array}\right]=\Phi_{1} \Phi_{1}^{*}+\Phi_{2} \Phi_{2}^{*}=A_{1} I_{N}+\Phi_{2} \Phi_{2}^{*}
$$

which implies that $\Phi_{2} \Phi_{2}^{*}=\left(A-A_{1}\right) I_{N}$. Consequently, $\Phi_{2}$ is tight frame with frame bound $A-A_{1}>0$.

For the second part, observe that if $\Phi$ is prime, there is nothing to prove. So suppose that $\Phi$ is divisible tight frame with frame bound $A$. Then, by definition, we can partition $\Phi$ into $\Phi=\Phi_{1} \cup\left(\Phi \backslash \Phi_{1}\right)$, where $\Phi_{1} \subsetneq \Phi$ is a tight frame. It follows from the proof of the first part of the lemma that $\Phi_{2}:=\Phi \backslash \Phi_{1}$ is also a tight frame. If $\Phi_{1}$ and $\Phi_{2}$ are prime, we are done. If not, repeat the process. In each step of this procedure we split a tight frame into two sets of cardinality at least $N$ each. Hence, by Remark 1, the procedure terminates after at most $\left\lfloor\frac{M}{N}\right\rfloor$ steps.

The second part of Theorem 2.3 suggests the following definition.

Definition 2.4. Let $\Phi$ be a tight frame. Suppose, for some $K \in \mathbb{N}$,

$$
\Phi=\Phi_{1} \cup \cdots \cup \Phi_{K}
$$

where $\Phi_{k}, k=1, \ldots, K$, are prime tight frames. We shall say that $\Phi_{k}$ are prime factors or prime divisors of $\Phi$.

The prime factors of a frame are, in general, not unique as shown by the following examples.

Example 1. (a) The 6 th roots of unity FUNTF in $\mathbb{R}^{2}$, that is, the frame of six unit-norm vectors each $2 \pi / 6$ apart as the vertices of a hexagon, factors into two FUNTFs, each of which consists of three vectors. But these are not unique since you can choose the two set of three frame vectors in eight different ways. However, up to multiplication of individual frame vectors by -1 this decomposition is in fact unique.

(b) The uniqueness of the factors up to scalar multiplication in part (a) of the example does not hold in general. The harmonic frame of 10 vectors in $\mathbb{C}^{2}$ decomposes into either five frames of size two or two frames of size five. We refer to Section 3.2 for more details on this. 
Tight frames $\Phi$ and $\Psi$ are unitarily equivalent if there is a bijection $p:\{1, \ldots, M\} \rightarrow$ $\{1, \ldots, M\}$, a unitary $N \times N$ matrix $U$ and a $c_{i} \in \mathbb{K}$ with $\left|c_{i}\right|=c>0$ such that $\psi_{i}=c_{i} U \varphi_{p(i)}$ for all $i=1, \ldots, M$. In matrix notation this reads $\Psi=U \Phi P C$, where $P$ is the $M \times M$ permutation matrix for $p$ and $C=\operatorname{diag}\left(c_{1}, \ldots, c_{M}\right)$. Note that there exist other related equivalence notions, e.g., in [8] $c_{i}$ is replaced by a fixed positive scalar $c>0$. We remark that it is only necessary to introduce permutations in the equivalence relations if one considers frames as sequences of vectors as opposed to non-ordered collections of vectors with repetition allowed. The following result shows that prime frame are equivalence classes.

Proposition 2.5. Suppose $\Phi$ and $\Psi$ are unitarily equivalent tight frames for $\mathbb{K}^{N}$. Then $\Phi$ is prime if and only if $\Psi$ is prime.

Proof. We prove that $\Phi$ is divisible if and only if $\Psi$ is divisible. Assume $\Phi_{J}:=\left[\varphi_{i}\right]_{i \in J}$ is a divisor of $\Phi$ for some $J \subset\{1, \ldots, M\}$. Then $\Phi_{J} \Phi_{J}^{*}=A I_{N}$ for some $A>0$. Since $\psi_{p^{-1}(i)}=$ $c_{p^{-1}(i)} U \varphi_{i}$ by assumption, we have that

$$
\Psi_{p^{-1}(J)}\left(\Psi_{p^{-1}(J)}\right)^{*}=U \Phi_{J} C_{J}\left(U \Phi_{J} C_{J}\right)^{*}=c^{2} A I_{N},
$$

where $C_{J}=\operatorname{diag}\left(c_{p^{-1}(i)}\right)_{i \in J}$. This shows that $\Psi$ is divisible with divisor $\Psi_{p^{-1}(J)}$. By the symmetry of the equivalence relation, this completes the proof.

Proposition 2.5 also holds for the notion of unitarily equivalence used in [8]. However, the result is false if one uses the coarser notion of equivalence, where the matrix $U$ is only assumed to be invertible.

We end this subsection by mentioning that the notion of prime tight frames easily generalizes to non-tight frames. Recall that if $\Phi$ is a frame with frame operator $S=\Phi \Phi^{*}$, then the associated canonical Parseval frame is given by $S^{-1 / 2} \Phi$. Hence, a frame $\Phi$ is said to be prime if its canonical Parseval frame is a prime tight frame.

\subsection{Existence and denseness of prime tight frames}

We now turn the question of existence of prime tight frames. If $\Phi$ is a union of an orthonormal basis for $\mathbb{K}^{N}$ and $M-N$ zero vectors, then $\Phi$ is prime for any $N, M \in \mathbb{N}$. This trivial observation shows the existence of prime tight frames for all $M \geq N \in \mathbb{N}$. The following result extends this fact to tight frames of non-zero vectors.

Proposition 2.6. For each dimension $N \in \mathbb{N}$ there exists a prime tight frame for $\mathbb{K}^{N}$ with $M$ non-zero vectors for any $M \geq N \in \mathbb{N}$.

Proof. The case $N=1$ is trivial, hence we assume $N \geq 2$. Let $\Psi \in \mathbb{K}^{(N-1) \times(M-1)}$ be a Parseval frame for $\mathbb{K}^{N-1}$. We now extend $\Psi$ into an $N \times M$ matrix by first adding a $1 \times(M-1)$ row vector with zeros as a new $N$ th row, and then by adding $e_{N} \in \mathbb{K}^{N}$ as a new $M$ th column. The picture is:

$$
\Phi=\left[\begin{array}{cccc}
\ddots & \vdots & . \cdot & 0 \\
\cdots & \Psi & \cdots & 0 \\
\cdot & \vdots & \ddots & 0 \\
0 & 0 & 0 & 1
\end{array}\right]
$$

The new frame $\Phi$ is a Parseval frame for $\mathbb{K}^{N}$ since $\Phi \Phi^{*}=I_{N}$. Moreover, it is prime since any tight frame $\Phi_{1} \subset \Phi$ must contain the $M$ th column in order to span $\mathbb{K}^{N}$. 
Remark 2. For certain values of $N, M$, we can extend Proposition 2.6 to show existence of prime unit-norm tight frames. The case $N \leq M<2 N$ follows from Remark 1 . If $M \geq 2 N$ and $M$ is prime, then we take the $M \times M$ DFT matrix and choose any $N$ rows to be our frame $\Phi$. Since no proper subset of the (primitive) $M$ th root of unity sum to zero, there exists no way to divide $\Phi$ into two FUNTFs. Hence, $\Phi$ is a prime FUNTF.

It is easy to build divisible tight frames since the union of any two tight frames is divisible. However, the existence of prime tight frames allows us to prove that "most" tight frame are in fact prime. To state this result, we need to set some notations. For $A>0$ fixed, let $\mathscr{F}(N, M, A)$ be the set of all tight frames with frame bound $A$, that is,

$$
\mathscr{F}(N, M, A)=\left\{\Phi \in \operatorname{Mat}(N \times M, \mathbb{K}): \Phi \Phi^{*}=A I_{N}\right\} .
$$

Let

$$
\mathscr{P}(N, M, A)=\{\Phi \in \mathscr{F}(N, M, A): \Phi \text { is prime }\} .
$$

When $A=1$, we simply denote these sets $\mathscr{F}(N, M)$ and $\mathscr{P}(N, M)$. Note that $\mathscr{F}(N, M)$ is the Stiefel manifold, see [16], which is invariant under multiplication by $N \times N$ unitary matrices from the left and by $M \times M$ unitaries from the right. Consequently, there exists an invariant Haar probability measure $\mu$ on $\mathscr{F}(N, M)$. The results stated below hold for any measure that is absolutely continuous with respect to $\mu$.

Following the setup in [1, Section 3], each of the entries in a matrix $\Phi \in \mathscr{F}(N, M)$ is written in terms of its real and imaginary parts: $\varphi_{k, \ell}=x_{k, \ell}+i y_{k, \ell}$ for all $k, \ell$. In this setting we can consider $\mathscr{F}(N, M)$ as an real algebraic variety in $\mathbb{R}^{2 M N}$. Since $\mathscr{F}(N, M)$ is an irreducible variety, every non-empty Zariski-open subset of $\mathscr{F}(N, M)$ is open and dense in $\mathscr{F}(N, M)$ in the (induced) standard topology [1]. Moreover, the complement of a non-empty Zariski-open subset is of $\mu$-measure zero. The following result says that being prime within the set of tight frames is a very generic property.

Theorem 2.7. Let $N, M \in \mathbb{N}$ and $A>0$. The set of prime A-tight frames $\mathscr{P}(N, M, A)$ is open and dense in the set of all $A$-tight frames $\mathscr{F}(N, M, A)$ in the (induced) standard topology. Moreover, the complement $\mathscr{F}(N, M, A) \backslash \mathscr{P}(N, M, A)$ is of measure zero in $\mu$.

Proof. We can without loss of generality take $A=1$. Let $\Phi \in \mathscr{F}(N, M)$ and let $S$ be the power set of $\{1, \ldots, M\}$. We use the notation $\Phi_{I}=\left[\varphi_{i}\right]_{i \in I}$ for $I \in S$. Now, $\Phi$ is divisible if and only if

$$
\Phi_{I} \Phi_{I}^{*}=c I_{N}
$$

for some $\emptyset \neq I \in S$ and $c>0$. These orthogonality conditions can be expressed as polynomial equations in $x_{k, \ell}$ and $y_{k, \ell}$ introduced above. Since $S$ is finite, the set of prime 1-tight frames $\mathscr{P}(N, M)$ is a finite intersection of the complement of such sets in $\mathscr{F}(N, M)$. The sub-variety $\mathscr{P}(N, M)$ is therefore Zariski-open in the irreducible variety $\mathscr{F}(N, M)$. By Proposition 2.6 the set $\mathscr{P}(N, M)$ is non-empty. Since $\mathscr{P}(N, M)$ is a non-empty Zariski-open set in an irreducible variety, the result follows.

Remark 3. Let $V$ be an $N \times M$ matrix with entries independently drawn at random from an absolutely continuous distribution with respect to the Lebesgue measure; a standard choice could be the Gaussian distribution of zero mean and unit variance. With probability one, $V$ is a frame and thus performing the Gram-Schmidt algorithm on the rows of $V$ leads to a tight frame. We call such frames for random, tight frames. It can be shown from Theorem 2.7 that random tight frames are prime with probability one. 
By Theorem 2.7 we see that if $\Phi$ is a (divisible or prime) tight frame and $\widetilde{\Phi}$ a random, arbitrarily small perturbation of $\Phi$ such that $\widetilde{\Phi}$ again is tight, then $\widetilde{\Phi}$ is prime with probability one. From Theorem 2.7 we also have the following density result.

Corollary 2.8. Every tight frame is arbitrarily close to a prime tight frame.

\section{Classification of certain prime tight frames}

In this section we characterize prime frames within three well-known families of FUNTFs: equiangular tight frames, harmonic frames, and spectral tetris frames.

\subsection{Equiangular FUNTFs}

A FUNTF $\Phi=\left\{\varphi_{i}\right\}_{i=1}^{M}$ is said to be equiangular if $\left|\left\langle\varphi_{i}, \varphi_{j}\right\rangle\right|=c$ for all $i, j=1, \ldots, M$ with $i \neq j$ for some constant $c \geq 0$, [17]. Equivalently, equiangular tight frames are sequences that achieve the Welch bounds with equality [18]. We show that, when they exist, equiangular tight frames are automatically prime tight frames. This necessary condition was derived by Xia, Zhou, and Giannikis [19] for the special case of harmonic tight frames using the notion of difference sets.

Theorem 3.1 ([17, Theorem 2.3]). Suppose $\Phi=\left\{\varphi_{i}\right\}_{i=1}^{M}$ be a unit norm frame in $\mathbb{K}^{N}$. Then

$$
\max _{i \neq j}\left|\left\langle\varphi_{i}, \varphi_{j}\right\rangle\right| \geq \sqrt{\frac{M-N}{N(M-1)}},
$$

and equality holds if and only if $\Phi$ is an equiangular tight frame.

The existence of equiangular tight frames is still an open problem. However, as shown below, when they exist, equiangular tight frames are also prime.

Theorem 3.2. Let $N \geq 2$. Equiangular FUNTFs of $M$ vectors in $\mathbb{K}^{N}$, when they exist, are prime.

Proof. Assume that $\Phi=\left\{\varphi_{i}\right\}_{i=1}^{M} \subset \mathbb{K}^{N}$ is an equiangular tight frame. Assume towards a contradiction that $\Phi$ is $(M, p)$-divisible for some $N \leq p \leq M-N$. Then write $\Phi=\Phi_{1} \cup \Phi_{2}$ where $\Phi_{1}$ and $\Phi_{2}$ are divisors of $\Phi$ of size $p$ and $M-p$, respectively. Consequently, we see that $\Phi$, $\Phi_{1}$, and $\Phi_{2}$ are all equiangular tight frames. According to Theorem 3.1,

$$
\left|\left\langle\varphi_{i}, \varphi_{j}\right\rangle\right|=\sqrt{\frac{M-N}{N(M-1)}}=\sqrt{\frac{p-N}{N(p-1)}}=\sqrt{\frac{M-p-N}{N(M-p-1)}}
$$

with $N \leq p \leq M-N$. But a series of easy calculations leads to a contradiction. Thus, $\Phi$ cannot be $(M, p)$-divisible for any $N \leq p \leq M-N$.

Note that Grassmanian tight frames, see [3], are not in general prime tight frames, e.g., any Grassmanian frame of four frame vectors in $\mathbb{R}^{2}$ is $(4,2)$-divisible. 


\subsection{Harmonic frames}

We now characterize all harmonic FUNTFs that are prime, and for those that are divisible we describe their factors. For this, we recall that given $M \geq N$, a harmonic tight frame (HTF) is obtained by keeping and renormalizing the $N$ first coordinates from an $M \times M$ discrete Fourier transform, that is, $\Phi=\left\{\varphi_{k}\right\}_{k=0}^{M-1}$ is a HTF, where the $(k+1)$ th column of $\Phi$ is given by

$$
\varphi_{k}=\sqrt{\frac{s}{N}}\left(\begin{array}{c}
1 \\
\gamma_{M}^{k} \\
\gamma_{M}^{2 k} \\
\vdots \\
\gamma_{M}^{(N-1) k}
\end{array}\right)=\sqrt{\frac{s}{N}}\left(\begin{array}{c}
\omega_{1}^{k} \\
\omega_{2}^{k} \\
\omega_{3}^{k} \\
\vdots \\
\omega_{N}^{k}
\end{array}\right) \in \mathbb{C}^{N}
$$

where $s>0, \gamma_{M}:=\exp (2 \pi i / M)$ is the $M$ th root of unity and $\omega_{n}:=\gamma_{M}^{n-1}=\mathrm{e}^{2 \pi i(n-1) / M}$ for $n=1, \ldots, N$. When there is no risk of confusion we shall simply write $\gamma$ for $\gamma_{M}=\exp (2 \pi i / M)$. We denote the obtained $\operatorname{HTF}$ by $\operatorname{HTF}(N, M, s)$, and we see that this tight frame has frame bound $A=s M / N$ and frame vector norms $\left\|\varphi_{k}\right\|=s$. Hence, for $s=1$ we have a unit-norm, $M / N$ tight frame, while we for $s=N / M$ have a Parseval frame. When nothing else is mentioned we assume $s=1$ for simplicity.

Let us fix some notations and assumptions for this section. We will always assume $N \geq 2$ since, if $N=1$, any HTF is $(M, p)$-divisible for all $p$. Throughout this section we will denote the index set $\{1,2, \ldots, M\}$ by $I$, and we will let $J_{1}$ denote a subset of $I$ and put $J_{2}:=I \backslash J_{1}$. If $d$ is a divisor of $M$, we define index sets $I(d, q) \subset I=\{1,2, \ldots M\}$ as follows

$$
I(d, q)=\left\{k \frac{M}{d}+q: k=0,1, \ldots, d-1\right\}, \quad q=1, \ldots, M / d .
$$

These index sets are a disjoint partition of $I=\{1,2, \ldots, M\}$ for a fixed divisor $d$. For any $n=$ $1,2, \ldots, N-1$, the set $\left\{\left(\gamma^{n}\right)^{m}: m \in I(d, 1)\right\}$ is a subgroup of $\left\{\left(\gamma^{n}\right)^{m}: m \in I\right\}$ in the circle group, and $\left\{\left(\gamma^{n}\right)^{m}: m \in I(d, q)\right\}$ is a coset. Furthermore, we assume the following prime factorization $M=p_{1}^{\alpha_{1}} p_{2}^{\alpha_{2}} \cdots p_{r}^{\alpha_{r}}$ with $\alpha_{i} \in \mathbb{N}$ and $p_{i}$ prime and ordered such that $p_{i}>p_{i+1}$.

Let $\Phi=\left\{\varphi_{k}\right\}_{k=1}^{M} \subset \mathbb{C}^{N}$ be a HTF with index set $I=\{1,2, \ldots, M\}$. Since $\Phi=\left\{\varphi_{k}\right\}_{k \in I}$ is a tight frame, the rows of $\Phi$ are equal-norm and orthogonal. In particular, we have

$$
0=\left\langle\varphi^{n}, \varphi^{n^{\prime}}\right\rangle=\sum_{m=1}^{M}\left(\gamma^{(n-1)-\left(n^{\prime}-1\right)}\right)^{m-1}=\sum_{m \in I}\left(\gamma^{n-n^{\prime}}\right)^{m-1} \quad \text { for } n \neq n^{\prime} \in\{1,2, \ldots, N\},
$$

where $\varphi^{j}$ denotes the $j$ th row of $\Phi$. Now, $\Phi$ is divisible exactly when it is possible to split the sum over $m \in I$ into two sums, each summing to zero, for each $n \neq n^{\prime}$. Here we have used that the norms of the $N$ rows of any sub-collection of $\Phi$ are automatically equal since the entries of $\Phi$ are equal in modulus. Therefore it is only necessary to consider the row-orthogonality requirement of potential divisors. We will use the following result repeatedly.

Lemma 3.3. Let $N, M \geq 2$ be given, and let $\Phi=\left\{\varphi_{k}\right\}_{k=1}^{M} \subset \mathbb{C}^{N}$ be a HTF. Then $\Phi$ is $(M, p)$ divisible if and only if there exists $J_{1} \subset I=\{1, \ldots, M\}$ with $\# J_{1}=p$ such that

$$
0=\sum_{m \in J_{1}}\left(\gamma^{n-1}\right)^{m-1} \quad \text { for } n \in\{2, \ldots, N\} .
$$


Proof. Let $\Phi_{1}:=\Phi_{J_{1}}=\left\{\varphi_{k}\right\}_{k \in J_{1}}$, and let $\varphi_{1}^{j}$ be the $j$ th row of $\Phi_{1}$. Note that the equations in (3.3) are equivalent to the statement that $\left\langle\varphi_{1}^{1}, \varphi_{1}^{n}\right\rangle=0$ for $n=2, \ldots, N$. Assume (3.3) holds. Then, since $\gamma^{-k}=\gamma^{M-k}$, we see that

$$
0=\sum_{m \in J_{1}}\left(\gamma^{n-n^{\prime}}\right)^{m-1}
$$

holds for all $n, n^{\prime} \in\{2, \ldots, N\}$ with $n \neq n^{\prime}$. The last statement is equivalent to $\left\langle\varphi_{1}^{n}, \varphi_{1}^{n^{\prime}}\right\rangle=0$ for all $n \neq n^{\prime} \in\{2, \ldots, N\}$ which in matrix notation becomes $\Phi_{1} \Phi_{1}^{*}=c I_{n}$. Therefore, equation (3.3) implies that $\Phi_{1}$ is a tight frame. The converse implication follows easily from the above.

Suppose $\Phi$ is $(M, p)$-divisible. By taking $n=2$ in (3.3) we see that the sub-sum over $J_{1}$ must be the sum of $p M$ th roots of unity, and, of course, the second sum over $J_{2}$ must be the sum of $(M-p) M$ th roots of unity. This is an example of vanishing sums of roots of unity [14]. When one of the vanishing sub-sums contains $p$ distinct $M$ th roots of unity, one says that $M$ is p-balancing [15]. In particular, we shall use the following result proved in [15].

Theorem 3.4 ([15, Theorem 2]). Let $M=p_{1}^{\alpha_{1}} p_{2}^{\alpha_{2}} \cdots p_{r}^{\alpha_{r}}$ with each $p_{i}$ prime and each $\alpha_{i}>0$. Then $M$ is $k$-balancing if and only if both $k$ and $M-k$ are in $\mathbb{N}_{0} p_{1}+\mathbb{N}_{0} p_{2}+\ldots+\mathbb{N}_{0} p_{r}$, that is, both $k$ and $M-k$ are linear combination of $p_{i}$ with nonnegative integer coefficients.

By Lemma 3.3, we immediately have that, for $N=2$, a HTF is $(M, p)$-divisible if and only if $M$ is $p$-balancing. More precisely, we have:

Corollary 3.5. Let $M \geq 2$ be given. Suppose $\Phi=\left\{\varphi_{k}\right\}_{k=1}^{M} \subset \mathbb{C}^{2}$ is a HTF. Then $\Phi$ is prime if and only if $M$ is a prime integer. Furthermore, if $M$ is not prime, then $\Phi$ is $(M, d)$-divisible for each $2 \leq d \leq M-2$ for which both $d$ and $M-d$ are in $\mathbb{N}_{0} p_{1}+\mathbb{N}_{0} p_{2}+\ldots+\mathbb{N}_{0} p_{r}$, in particular, $\Phi$ is $(M, d)$-divisible for every divisor $d$ of $M$.

Proof. We will use the fact that the frame $\operatorname{HTF}(2, M, s)$ is prime if and only if $M$ is not $d$ balancing for any $2 \leq d \leq M-2$.

Assume first that $\Phi$ is prime, that is, that $M$ is not $d$-balancing for any $2 \leq d \leq M-2$. Towards a contradiction assume further that $M$ is not prime so that $M=p_{1}^{\alpha_{1}} p_{2}^{\alpha_{2}} \cdots p_{r}^{\alpha_{r}}$ with $r>1$. Then $M=p_{1} b$ with $b=p_{1}^{\alpha_{1}-1} p_{2}^{\alpha_{2}} \cdots p_{r}^{\alpha_{r}} \geq 2$. For $d=p_{1}$ we have

$$
M-d=p_{1} b-p_{1}=p_{1}(b-1),
$$

but this contradicts the fact that $M$ is not $d$-balancing for any $2 \leq d \leq M-2$.

For the opposite implication, we observe that the prime factorization of $M=p_{1}$ is trivial when $M$ itself is prime. Since there is no divisor $0<d<M$ so that $d, M-d \in M \mathbb{N}_{0}$, we see that $M$ is not $d$-balancing for any $N \leq d \leq M-N$. Thus, we have proved the bi-implication part of the theorem.

The "furthermore" statement follows immediately from the above and Theorem 3.4.

In general, when $N>2$, the characterization of prime HTFs is more involved. Indeed, we now have multiple rows consisting of $M$ th roots of unity, and we must insure, for $(M, d)$ divisibility, not only that each of these row is $d$-balancing, but also that the subset of $d$ roots that sum to zero in each of these rows, comes from the same columns. 
In order to formally state this we need to define a few sets of integers. Using the notation fixed above, we define

$$
\begin{aligned}
D_{M, N} & =\{d \in\{N, \ldots, M-N\}: d \text { is a divisor of } M\}, \\
P_{M, N} & =D_{M, N} \backslash\left\{d \in D_{M, N}: \exists c \in D_{M, N} \text { such that } c \text { is a divisor of } d\right\},
\end{aligned}
$$

and

$$
S_{M, N}=\left\{s \in\{N, \ldots, M-N\}: s=\sum_{k=1}^{K} a_{k} q_{k}, M-s=\sum_{k=1}^{K} b_{k} q_{k}, \text { where } a_{k}, b_{k} \in \mathbb{N}_{0}, q_{k} \in P_{M, N}\right\},
$$

where $K=\# P_{M, N}$. Note that $s \in S_{M, N}$ if and only if $M-s \in S_{M, N}$. It is also clear that

$$
P_{M, N} \subset D_{M, N} \subset S_{M, N},
$$

and that $D_{M, N}$ is empty for any $N \in \mathbb{N}$ if $M$ is prime or if $M<2 N$. Note that if $M \geq 2 N$, the condition $d \leq M-N$ in the definition of $D_{M, N}$ is redundant as no divisor of $M$ can be greater than $M-N$. Moreover, the set $S_{M, N}$ is empty, if and only if $D_{M, N}$ is empty. The significance of these sets will become evident in Theorems 3.6 and 3.8 and Corollary 3.7 below, but we mention here that $P_{M, N}$ will determine the cardinality of the prime factors and $S_{M, N}$ the cardinality of every possible divisor of the HTF. In the following example we calculate these sets for various $M, N \in \mathbb{N}$.

Example 2. (a) For prime $M \in \mathbb{Z}$ and any $N \in \mathbb{N}$, we have $P_{M, N}=D_{M, N}=S_{M, N}=\emptyset$.

(b) For $M=9, N=2$ or $N=3: D_{M, N}=P_{M, N}=\{3\}, S_{M, N}=\{3,6\}$.

(c) For $M=9, N \geq 4$ : $D_{M, N}=P_{M, N}=S_{M, N}=\emptyset$.

(d) For $M=10, N=2: D_{M, N}=P_{M, N}=\{2,5\}, S_{M, N}=\{2,4,5,6,8\}$.

(e) For $M=10, N=3,4$ or 5: $D_{M, N}=P_{M, N}=S_{M, N}=\{5\}$.

(f) For $M=24, N=2: D_{M, N}=\{2,3,4,6,8,12\}, P_{M, N}=\{2,3\}, S_{M, N}=\{2,3, \ldots, 22\}$.

(g) For $M=24, N=3: D_{M, N}=\{3,4,6,8,12\}, P_{M, N}=\{3,4\}, S_{M, N}=\{3, \ldots, 21\} \backslash\{5,19\}$.

(h) For $M=24, N=4$ : $D_{M, N}=\{4,6,8,12\}, P_{M, N}=\{4,6\}, S_{M, N}=\{4,6, \ldots, 20\}$.

Given $d \in \mathbb{N}$, the following well-known fact will be used repeatedly:

$$
\frac{1}{d} \sum_{k=0}^{d-1} \mathrm{e}^{2 \pi i \frac{k m}{d}}= \begin{cases}1 & m \in d \mathbb{Z} \\ 0 & m \in \mathbb{Z} \backslash d \mathbb{Z} .\end{cases}
$$

We now state and prove the following result characterizing all HTFs that are prime.

Theorem 3.6. Let $N, M \in \mathbb{N}$ and $s>0$ be given, and let $\Phi=\left\{\varphi_{k}\right\}_{k=1}^{M}=\operatorname{HTF}(N, M, s) \subset \mathbb{C}^{N}$. Then $\Phi$ is prime if and only if $D_{M, N}=\emptyset$. In particular, if $M$ is prime, then $\Phi$ is prime.

Proof. Suppose that $\Phi$ is prime and let us prove that $D_{M, N}=\emptyset$. Assume by way of contradiction that there exists $d \in D_{M, N} \neq \emptyset$, i.e., $d \geq N$ is a divisor of $M$. Take $J_{1}=I(d, 1)$, where $I(d, 1)$ is defined in (3.1). Then, for $n \in\{2, \ldots, d\}$,

$$
\sum_{m \in J_{1}}\left(\gamma_{M}^{n-1}\right)^{m-1}=\sum_{k=0}^{d-1} \mathrm{e}^{\frac{2 \pi i}{M}(n-1)\left(k \frac{M}{d}\right)}=\sum_{k=0}^{d-1} \mathrm{e}^{2 \pi i \frac{k(n-1)}{d}}=0,
$$


where the last equality follows from (3.6). Since $d \geq N$, we see by Lemma 3.3 that $\Phi$ is $(M, d)$ divisible with divisor $\Phi_{1}:=\Phi_{J_{1}}$ which is a contradiction.

We now prove that if $\Phi$ is not prime, then $D_{M, N} \neq \emptyset$. Thus assume that $\Phi$ is $(M, d)$-divisible for some $d$ such that $N \leq d \leq M-N$. Let $\Phi=\Phi_{1} \cup \Phi_{2}$ be divisors of $\Phi$, where $\Phi_{1}=\Phi_{J_{1}}$ and $\Phi_{2}=\Phi_{J_{2}}$ with $\# J_{1}=d$ and $\# J_{2}=M-d$. By Lemma 3.3, we see that our assumption is equivalent to assuming the existence of a index set $J_{1} \subset\{1, \ldots, M\}$ of cardinality $d$ such that

$$
\sum_{m \in J_{1}}\left(\gamma_{M}^{n-1}\right)^{m-1}=\sum_{m \in J_{1}^{n}} \gamma_{M}^{m-1}=0
$$

holds for each $n=2, \ldots, N$, where $J_{1}^{n}:=(n-1) J_{1} \bmod M$. In particular, for $n=2$, this means that $M$ is $d$-balancing, hence $d=\sum_{k=1}^{r} a_{k} p_{k}$ and $M-d=\sum_{k=1}^{r} b_{k} p_{k}$ for $a_{k}, b_{k} \in \mathbb{N}_{0}$. Let $R_{1} \subset$ $\{1, \ldots, r\}$ be the indices $k$ for which $a_{k} \neq 0$. We see that the index set $J_{1}$ has the following form

$$
J_{1}=\bigcup_{k \in R_{1}} \bigcup_{q_{k} \in Q_{k}} I\left(p_{k}, q_{k}\right)=\bigcup_{k \in R_{1}} I_{k}, \quad \text { where } I_{k}:=\bigcup_{q_{k} \in Q_{k}} I\left(p_{k}, q_{k}\right),
$$

for some $Q_{k} \subset\left\{1, \ldots, M / p_{k}\right\}$ with $\# Q_{k}=a_{k}$ for each $k \in R_{1}$. We can assume without loss of generality that, for $i, j \in R_{1}$,

$$
n_{i} p_{i} \neq n_{j} p_{j} \quad \text { for each } n_{i}=1, \ldots, a_{i} \text { and } n_{j}=1, \ldots, a_{j},
$$

whenever $i \neq j$.

We need to prove that $D_{M, N} \neq \emptyset$. It follows from $d=\sum_{k \in R_{1}} a_{k} p_{k}$ that, if $p_{k_{0}} \geq N$ for some $k_{0} \in R_{1}$, then $p_{k_{0}} \in D_{M, N} \neq \emptyset$, which concludes the proof. If however, $p_{k}<N$ for each $k \in R_{1}$, we claim that $a_{k} p_{k} \geq N$ for each $k \in R_{1}$. To show this claim, let $k_{0} \in R_{1}$ be fixed and assume $p_{k}<N$ for all $k \in R_{1}$. Since $p_{k}<N$ is prime, it follows that

$$
\sum_{m \in I_{k}}\left(\gamma_{M}^{(n-1)}\right)^{m-1}=0
$$

for $n=2, \ldots, p_{k}, p_{k}+2, \ldots$ for $k \in R_{1}$. We remark that $p_{k_{0}}$ is not a $n_{j}$-multiple of any of the other prime numbers $p_{j}$ for $n_{j}=1, \ldots, a_{j}$ by equation (3.8) which, in turn, implies that

$$
\sum_{m \in J_{1} \backslash I_{k_{0}}}\left(\gamma_{M}^{p_{k_{0}}}\right)^{m-1}=0
$$

for $n=p_{k_{0}}+1$. Hence, it follows by the fact that $\Phi_{1}$ is a tight frame, that

$$
\sum_{m \in I_{k_{0}}}\left(\gamma_{M}^{p_{k_{0}}}\right)^{m-1}=p_{k} \sum_{q \in Q_{k_{0}}}\left(\gamma_{M}^{p_{k_{0}}}\right)^{q-1}=0
$$

The last equality is only possible if the index set $Q_{k_{0}}$ is of a certain size, in particular, $\# Q_{k_{0}} \geq 2$ must be a divisor of $M / p_{k_{0}}$. If $M / p_{k_{0}}$ is prime, then $a_{k_{0}}=\# Q_{k_{0}}=M / p_{k_{0}}$ which is impossible. Hence, $M / p_{k_{0}}$ is not prime. Let $d_{1}$ be the smallest divisor of $M / p_{k_{0}}$. By (3.9) we then see that $\# Q_{k_{0}}=n d_{1}$ for some $n \in \mathbb{N}$ since $Q_{k_{0}}$ must be a union of $n$ sets, say $D_{\ell}^{1}, \ell=1, \ldots, n$, of cardinality $d_{1}$. If $d_{1} p_{k_{0}}>N$, we are done. If not, we consider row $n=d_{1} p_{k_{0}}+1$ in (3.7). Repeating the argument above leads to

$$
\sum_{q \in Q_{k_{0}}}\left(\gamma_{M}^{d_{1} p_{k_{0}}}\right)^{q-1}=0
$$


Note that $\left(\gamma_{M}^{d_{1} p_{k_{0}}}\right)^{q-1}$ is the same $M$ th root for all $q \in D_{\ell}^{1}$ when $\ell$ is fixed. If $M /\left(d_{1} p_{k_{0}}\right)$ is prime, then, by (3.9) and (3.10), $\# Q_{k_{0}}=M / p_{k_{0}}$ which is impossible. Hence, $M /\left(d_{1} p_{k_{0}}\right)$ is not prime, and we let $d_{2}$ be the smallest divisor of $M /\left(d_{1} p_{k_{0}}\right)$. By (3.9) and (3.10) we then see that $\# Q_{k_{0}}=n d_{1} d_{2}$ for some $n \in \mathbb{N}$. We can continue the argument which proves the claim.

By the proof of the claim, we also see that $a_{k_{0}} p_{k_{0}}$ is a divisor of $M$. Since we just proved $a_{k_{0}} p_{k_{0}}>N$, we arrive at the conclusion $a_{k_{0}} p_{k_{0}} \in D_{M, N} \neq \emptyset$.

The proof of the last statement in Theorem 3.6 is an easy consequence of the fact that if $M$ is prime, then $D_{M, N}=\emptyset$.

Remark 4. (a) The last part of the above result states that a HTF $\Phi=\left\{\varphi_{k}\right\}_{k=1}^{M} \subset \mathbb{C}^{N}$ with $M \geq 2 N$ is prime if $M$ is prime. The converse of this is not true. Indeed, consider the HTF with $M=9$ and $N=4$, see Example 2(c). This frame is prime, but $M$ is not.

(b) Theorem 3.4 classifies all $k$ for which $M$ is $k$-balancing, but it does not give how to choose the $k$ distinct roots out of the $M$ roots of unity. What the proof of Theorem 3.6 shows is that for a HTF to be $(M, d)$-divisible, in addition to having a vanishing sub-sum of $d$ distinct $M$ th roots of unity, we must also ensure that the sub-sum of every $n$th power of these roots of unity vanishes for $n=2, \ldots, N-1$. We refer to Corollary 3.7 below for a statement on how to choose the $d$ distinct roots of unity so that all these sub-sums vanishes.

In case the HTF is divisible i.e., $D_{M, N} \neq \emptyset$, the following result tells us how to factor it into prime divisors. The proof follows from the proof of Theorem 3.6.

Corollary 3.7. Let $N, M \in \mathbb{N}$ and $s>0$ be given, and let $\Phi=\left\{\varphi_{k}\right\}_{k=1}^{M}=\operatorname{HTF}(N, M, s) \subset \mathbb{C}^{N}$. Suppose that $D_{M, N} \neq \emptyset$ and $d<M$. Then the following assertions are equivalent:

(i) $d \in P_{M, N}$,

(ii) $\Phi$ factors into $M / d$ prime FUNTFs each of cardinality $d$, that is,

$$
\Phi=\bigcup_{i=1}^{M / d} \Phi_{i}
$$

with $\Phi_{i}$ being prime and $\# \Phi_{i}=d$.

Furthermore, one of the factors from assertion (ii), say $\Phi_{1}$, is the HTF with d frame vectors is $\mathbb{C}^{N}$, that is, $\Phi_{1}=\operatorname{HTF}(N, d, s)$. Let $U=\operatorname{diag}\left(\gamma^{0}, \gamma^{1}, \ldots, \gamma^{N-1}\right) \in U(N)$, where $\gamma=\exp (2 \pi i / M)$. The other factors are then given as $\Phi_{i}=U^{i-1} \Phi_{1}$ for $i=1, \ldots, M / d$.

By Corollary 3.7 we know exactly how the prime "building blocks" of a divisible HTF look, hence we can use this structure to build larger divisors of the HTF. Suppose $\Phi$ is a HTF and $d \in D_{M, N} \neq \emptyset$. Then $\Phi$ is $(M, d)$-divisible, and, moreover, $\left\{\varphi_{i}\right\}_{i \in I(d, q)}$ is a tight frame for any $q=1, \ldots, M / d$. Now, we can combine these $M / d$ tight frames of cardinality $d$ into tight frames of cardinality $d, 2 d, 3 d, \ldots, M$. Hence, $\Phi$ is also $(M, n d)$-divisible for $n=1, \ldots, M / d-1$. Assume further that $M$ has another divisor which are also greater than $N$, say $\tilde{d} \in D_{M, N}$. We can then combine unions of $\left\{\varphi_{i}\right\}_{i \in I(d, q)}$ with unions of $\left\{\varphi_{i}\right\}_{i \in I(\tilde{d}, \tilde{q})}$, where $q=1, \ldots, M / d$ and $\tilde{q}=1, \ldots, M / \tilde{d}$, respectively, as long as the index sets are disjoint. Hence, to combine tight frames from different divisors, we only need to make sure that the same frame element $\varphi_{i}$ does not appear in both frames. We make these observations precise in the following result. 
Theorem 3.8. Let $M \geq N \geq 2$, and $\Phi=\left\{\varphi_{k}\right\}_{k=1}^{M} \subset \mathbb{C}^{N}$ be a HTF. If $D_{M, N} \neq \emptyset$, then $\Phi$ is $(M, s)$-divisible for each $s \in S_{M, N}$.

Proof. If $D_{M, N} \neq \emptyset$, then $\Phi$ is $(M, d)$-divisible for any divisor $d$ of $M$ such that $N \leq d \leq M-N$. We now show that $\Phi$, in fact, is $(M, s)$-divisible for each $s \in S_{M, N}$. By symmetry of the set $S_{M, N}$, it suffices to show that $\Phi$ is $(M, s)$-divisible for $s \in S_{M, N}$ with $s \leq M / 2$. For $s \in S_{M, N}$ we have $s=\sum_{k=1}^{K} a_{k} q_{k}$ with $a_{k} \in \mathbb{N}_{0}, q_{k} \in P_{M, N}$ and $K=\# P_{M, N}$. For each $k=1,2, \ldots k$ construct an $N \times q_{k}$ matrix $\Phi_{\ell}^{\left(p_{k}\right)}$ based on the first $q_{k}$ th roots of unity. By shifting this matrix $a_{k}-1$ times, we will have a collection of $a_{k}$ such matrices. Next define

$$
\Phi_{k}=\left[\begin{array}{llll}
\Phi_{1}^{\left(q_{k}\right)} & \Phi_{2}^{\left(q_{k}\right)} & \ldots & \Phi_{a_{k}}^{\left(q_{k}\right)}
\end{array}\right]
$$

Now $\Phi_{k}$ is an $N \times a_{k} q_{k}$ matrix whose rows are mutually orthogonal. We then obtain an $N \times d$ matrix

$$
\widetilde{\Phi_{1}}=\left[\begin{array}{llll}
\Phi_{1} & \Phi_{2} & \cdots & \Phi_{r}
\end{array}\right]
$$

which is a FUNTF. Hence, $\Phi$ is $(M, s)$-divisible.

Casazza and Kovačević introduced the notion of generalized harmonic frames in [7] and showed that these frames are unitarily equivalent to HTFs. Consequently, by Proposition 2.5, the results obtained in this section for HTFs extend to a classification of prime and divisible generalized HTFs.

\subsection{Computational aspects of harmonic tight frames}

For harmonic tight frames the prime building blocks are exactly described by Corollary 3.7. We wish to suggest a strategy that can be used to design efficient numerical tools for signal processing with divisible HTFs. Recall that, in analyzing a signal $x \in \mathbb{C}^{N}$ with any type of frame, one needs to compute $c=\left\{\left\langle x, \varphi_{i}\right\rangle\right\}_{i=1}^{M}$. A naïve way of computing the analysis step for divisible harmonic tight frames would be to zero pad $x \in \mathbb{C}^{N}$ into a vector $\hat{x} \in \mathbb{C}^{M}$ and then compute a full FFT of $\hat{x}$ of size $M$. However, Corollary 3.7 suggests a more effective analysis (and synthesis) process. Let $\Phi=\left\{\varphi_{i}\right\}_{i=1}^{M}$ be a divisible HTF. Suppose $p \in P_{M, N}$, where the set $P_{M, N}$ is defined by (3.5). The indices $I_{q}:=I(p, q)=\{k M / p+q: k=0,1, \ldots, p-1\}$ for each $q=1, \ldots, M / p$ are then the index sets of the $M / p$ prime factors $\Phi_{I_{q}}:=\left\{\varphi_{i}\right\}_{i \in I_{q}}$. By Corollary 3.7 these prime factors are closely related, in fact, $\Phi_{I_{q}}=U^{q-1} \Phi_{I_{1}}$, where $U=\operatorname{diag}\left(\gamma^{0}, \gamma^{1}, \ldots, \gamma^{N-1}\right) \in \mathrm{U}(N)$ is a unitary, diagonal matrix and $\gamma$ the $M$ th root of unity. Note that $\Phi_{I_{1}}$ is first $N$ rows of a $p \times p$ discrete Fourier matrix (up to scaling). Therefore, the analysis process of computing $\left\{\left\langle x, \varphi_{i}\right\rangle\right\}_{i=1}^{M}$ for some signal $x \in \mathbb{C}^{N}$ can be performed by first computing $y_{q}:=\left(U^{*}\right)^{q-1} x \in \mathbb{C}^{N}$ for each $q=1, \ldots, M / p$, then computing $c_{I_{q}}:=\Phi_{I_{1}}^{*} y_{q} \in \mathbb{C}^{p}$ for $q=1, \ldots, M / p$, and finally combining $c_{I_{q}}$ for each $q=1, \ldots, M / p$ into $c$. The first step $\left(U^{*}\right)^{q-1} x$ is fast and stable since $U$ a is diagonal unitary, the second step is $M / p$ FFTs of size only $p$, and the last step is simply a rearrangement of the coefficients. The number of operations needed in this decomposition strategy is of the order of $M \log _{2} p$ which should be compared to $O\left(M \log _{2} M\right)$ operations for the naïve strategy. However, more importantly, this decomposition can be implemented as a parallel algorithm for each $q=1, \ldots, M / p$, where we only have $O\left(p \log _{2} p\right)$ operations on each processor; this will lead to a significant speed-up in the analysis step in multi-core and multiprocessing systems. A similar speed-up procedure can be used for the synthesis process. 
In addition, recall that the worst-case coherence of a FUNTF $\Phi$ is given by

$$
\mu_{\Phi}=\max _{k \neq \ell}\left|\left\langle\varphi_{k}, \varphi_{\ell}\right\rangle\right|
$$

Given $M \geq N$, the coherence of $\Phi=\operatorname{HTF}(N, M, 1)$ is easily computed as

$$
\mu_{\Phi}=\frac{1}{N} \max _{k \neq \ell}\left|\sum_{n=0}^{N-1} \gamma_{M}^{n(k-\ell)}\right|=\frac{1}{N} \frac{\sin (\pi N / M)}{\sin (\pi / M)}=: \mu_{N, M}
$$

since the maximum is obtained for $|k-\ell|=1$. If $\Phi=\operatorname{HTF}(N, M, 1)$ is divisible, then for each $p \in P_{M, N}$, each of its $M / p$ prime HTFs have the same coherence $\mu_{1}$, i.e.,

$$
\mu_{1}=\mu_{N, p}=\frac{1}{N} \frac{\sin (\pi N / p)}{\sin (\pi / p)}
$$

which clearly satisfies $\mu_{1} \leq \mu_{\Phi}$. In fact, we always have $\mu_{1}<\mu_{\Phi}$ if $P_{M, N} \neq \emptyset$. Clearly, as the redundancy grows, i.e., $M \rightarrow \infty$ with $N$ fixed, we see that $\mu_{\Phi} \nearrow 1$. Thus decomposing divisible HTFs in their prime factors, results in these divisors having smaller coherence, e.g., if $p=d N$, we see that $\mu_{1} \searrow d \sin (\pi / d) / \pi$ as $N \rightarrow \infty$. Moreover, for the typical range $N \leq p \leq 2 N$, we see that $\mu_{1} \leq 2 / 3$ for any $N \geq 3$.

\subsection{Spectral Tetris frames}

Recently, Casazza, Fickus, Mixon, Wang, and Zhou [5] introduced the spectral tetris method as a mean to construct FUNTFs for $\mathbb{R}^{N}$ for any given $N, M \in \mathbb{N}$ satisfying $M \geq 2 N$. One of the key features of this class of frames is that they are sparse in the sense that the coefficient vector of each frame element with respect to an orthonormal basis contains only few nonzero entries [4]. For example, when $N=4$ and $M=11$, the spectral tetris construction yields the frame $\Phi=\left\{\varphi_{i}\right\}_{\in I}$ with $I=\{1,2, \ldots, 11\}$ :

$$
\Phi=\left[\begin{array}{ccccccccccc}
1 & 1 & \sqrt{\frac{3}{8}} & \sqrt{\frac{3}{8}} & 0 & 0 & 0 & 0 & 0 & 0 & 0 \\
0 & 0 & \sqrt{\frac{5}{8}} & -\sqrt{\frac{5}{8}} & 1 & \sqrt{\frac{1}{4}} & \sqrt{\frac{1}{4}} & 0 & 0 & 0 & 0 \\
0 & 0 & 0 & 0 & 0 & \sqrt{\frac{3}{4}} & -\sqrt{\frac{3}{4}} & 1 & \sqrt{\frac{1}{8}} & \sqrt{\frac{1}{8}} & 0 \\
0 & 0 & 0 & 0 & 0 & 0 & 0 & 0 & \sqrt{\frac{7}{8}} & -\sqrt{\frac{7}{8}} & 1
\end{array}\right],
$$

where the frame vectors of $\Phi$ appears as columns in the matrix. Observe that the first two vectors are identical, which might be an undesirable for encoding because they lead to transform coefficients $\left\langle f, \varphi_{i}\right\rangle$ carrying no new information [10]. Since $E_{4}=\left\{\varphi_{i}\right\}_{i \in I_{1}}, I_{1}=\{1,5,8,11\}$, is an orthonormal basis, we see that $\Phi_{1}=\Phi \backslash E_{4}$ is a tight frame with redundancy reduced to $7 / 4$, and this can not be reduced further if tightness should be preserved. The reduced tight frame $\Phi_{2}$ does not have the artifact of $\Phi$ with respect to repetition of vectors. Moreover, it is a tight frame with redundancy $M / N$ less than two, something that is not possible using spectral tetris algorithms from $[4,5]$. Hence, this reduced tight frame possesses additional desirable properties as compared to the original tight frame, without loosing the sparsity of the original frame $\Phi$.

We prove below that all spectral tetris tight frames can be decomposed similarly, and we characterize all prime spectral tetris frames. This characterization ultimately allows us to determine precisely when the spectral tetris construction works for $M / N \leq 2$ (Corollary 3.10). 
Before proving our main results we recall how the spectral tetris method works. Given any $N, M \in \mathbb{N}$ satisfying $M \geq 2 N$, let $\lambda=M / N$. The method was developed in [5] and extended in [4]. Here, we shall consider spectral tetris frames constructed from the algorithms in $[4,5]$ under the usual sparsity setup that the "tetris blocks" are of size $1 \times 1$ and $2 \times 2$.

We define $K=\left\{k_{n}: n=0,1, \ldots, \operatorname{gcd}\{N, M\}\right\}$, where $k_{n}=\frac{n N}{\operatorname{gcd}\{N, M\}}$. For $N, M \in \mathbb{N}$ such that $\lambda=M / N \geq 2$ the spectral tetris frame $\operatorname{STF}(N, M)$ in $\mathbb{R}^{N}$ is then given as the $M$ columns of

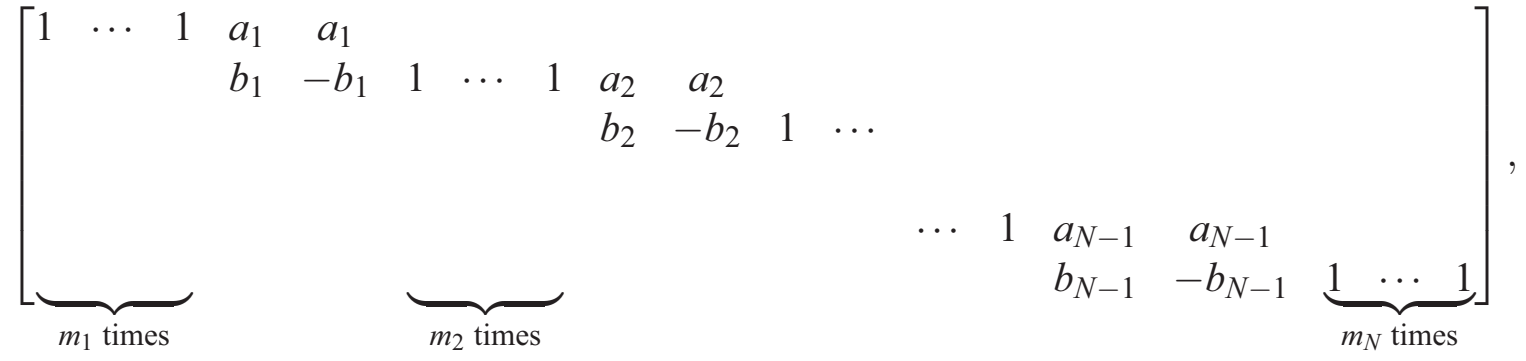

where $a_{j}:=\sqrt{\frac{r_{j}}{2}}$ and $b_{j}:=\sqrt{1-\frac{r_{j}}{2}}$, and $r_{j} \in[0,1)$ and $m_{j} \in \mathbb{N}_{0}, j=1, \ldots, N$ are defined by

$$
\begin{array}{llrl}
\lambda & =m_{j}+r_{j}, & & \text { when } j-1 \in K, \\
\lambda & =\left(2-r_{j-1}\right)+m_{j}+r_{j}, & & \text { otherwise. }
\end{array}
$$

If $j \in K$, the $2 \times 2$-block matrix $B_{j}=\left[\begin{array}{cc}a_{j} & a_{j} \\ b_{j} & -b_{j}\end{array}\right]$ is left out. Note that $r_{j}=0$ exactly when $j \in K$.

The following result characterizes prime spectral tetris tight frames.

Theorem 3.9. Let $N, M \in \mathbb{N}$ such that $\lambda:=M / N \geq 2$ be given. Suppose $\Phi$ is a spectral tetris FUNTF of $M$ vectors in $\mathbb{R}^{N}$ associated with $\lambda$. Then, either $\Phi$ is prime, or $\Phi$ factors as

$$
\Phi=\Phi_{1} \cup \bigcup_{l=1}^{L} E_{N},
$$

where $\Phi_{1}$ is a prime FUNTF of $M-L N$ vectors and $E_{N}=\left\{e_{j}\right\}_{j=1}^{N}$ is the standard orthonormal basis for $\mathbb{R}^{N}$. Moreover, $\Phi$ is divisible exactly when the eigenvalue $\lambda$ of the frame operator of $\Phi$ satisfy

$$
j \lambda-\lfloor(j-1) \lambda\rfloor \geq 3 \quad \text { for all } 1<j<\frac{N}{\operatorname{gcd}(N, M)} .
$$

In particular, $\Phi$ is divisible if $\lambda \geq 3-\frac{1}{N}$, i.e., $M \geq 3 N-1$.

Proof. Let $\left\{e_{j}\right\}_{j=1}^{N}$ denote the standard orthonormal basis of $\mathbb{R}^{N}$, and let $\Phi$ be a spectral tetris frame. We will use the notation introduced above. We claim that $\Phi$ is divisible if and only if $e_{j} \in \Phi$ for all $j=1, \ldots, N$. If $e_{j} \in \Phi$ for all $j=1, \ldots, N$, then $\Phi \backslash\left\{e_{j}\right\}_{j=1}^{N}$ and $\left\{e_{j}\right\}_{j=1}^{N}$ are tight frames hence $\Phi$ is divisible. This shows one direction of the claim. Now, assume that $e_{j_{0}} \notin \Phi$ for some $j_{0}=1, \ldots, N$. In other words, we assume that $m_{j_{0}}=0$. We consider the two $2 \times 2$ blocks $B_{j_{0}-1}$ and $B_{j_{0}}$. Let $i_{0}+l \in\{1, \ldots, M\}$ for $l=0,1,2,3$ be the indices of the columns of $\Phi$ associated with $B_{j_{0}-1}$ and $B_{j_{0}}$. Let $\varphi^{j_{0}}$ denote the $j_{0}$ th row of $\Phi$. Assume towards a contradiction that $\Phi=\Phi_{1} \cup \Phi_{2}$ is divisible, where $\Phi_{1}=\left\{\varphi_{i}\right\}_{i \in I_{1}}$ and $\Phi_{1}=\left\{\varphi_{i}\right\}_{i \in I_{2}}$ are tight frames. The common support of the rows $\varphi^{j_{0}}$ and $\varphi^{j_{0}+1}$ is $\left\{i_{0}+2, i_{0}+3\right\}$. Therefore, owing 
to orthogonality requirements of the rows, the frame vectors $\varphi_{i_{0}+2}$ and $\varphi_{i_{0}+3}$ need to belong to the same divisor, say $\Phi_{1}$. This in turn means that $\varphi_{i_{0}}, \varphi_{i_{0}+1} \in \Phi_{2}$ since $\Phi_{2}$ otherwise cannot span $\mathbb{R}^{N}$. The square norm of the $j_{0}$ th row of $\Phi_{1}$ is $a_{j_{0}}^{2}+a_{j_{0}}^{2}=r_{j_{0}}$, and the square norm of the $\left(j_{0}+1\right)$ th row of $\Phi_{1}$ is at least $b_{j_{0}}^{2}+\left(-b_{j_{0}}\right)^{2}=2-r_{j_{0}}$. Since $\Phi_{1}$ has equal row norm, this implies that $r_{j_{0}} \geq 2-r_{j_{0}}$, that is, $r_{j_{0}} \geq 1$ which contradicts $r_{j_{0}} \in[0,1)$. Hence, $\Phi$ is prime. This completes the proof of the claim.

In [13] it is shown that $e_{j} \in \Phi$ for all $j=1, \ldots, N$ if and only if

$$
\left(j_{0}-k_{n}\right) \lambda-\left\lfloor\left(j_{0}-1-k_{n}\right) \lambda\right\rfloor \geq 3
$$

for any $j_{0}$ such that $k_{n}+1<j_{0}<k_{n+1}$ for all $n=0,1, \ldots, \operatorname{gcd}\{N, M\}$. However, if (3.15) is satisfied for one $n=0,1, \ldots, \operatorname{gcd}\{N, M\}$, then it is satisfied for all $n=0,1, \ldots, \operatorname{gcd}\{N, M\}$. Hence, $e_{j} \in \Phi$ for all $j=1, \ldots, N$ if and only if

$$
j_{0} \lambda-\left\lfloor\left(j_{0}-1\right) \lambda\right\rfloor \geq 3
$$

for all $j_{0}$ satisfying $1=k_{0}+1<j_{0}<k_{1}=N / \operatorname{gcd}\{N, M\}$.

The prime FUNTF $\Phi_{1}$ from Theorem 3.9 is actually the output of the spectral tetris algorithm with $M-L N$ vectors. The frame bound of $\Phi_{1}$ is $\lambda_{1}=\frac{M-L N}{N}=\lambda-L$, which might be strictly smaller than two. It is not difficult to see that the spectral tetris construction always works when $M \geq 2 N-1$, but it is in general difficult to see for which $M$ and $N$ the construction still works when $N<M<2 N-1$. The next result characterizes when this is indeed possible. We denote the output of the spectral tetris algorithm $\operatorname{STF}(N, M)$ for all $N, M \in \mathbb{N}$. If the spectral tetris does not work for the given $N$ and $M$, we set $\operatorname{STF}(N, M)=\emptyset$.

Corollary 3.10. Let $N, \tilde{M} \in \mathbb{N}$ be given such that $N<\tilde{M}<2 N$. Define $M=\tilde{M}+N$ and $\lambda=$ $M / N \geq 2$. Then $\operatorname{STF}(N, \tilde{M})$ is a FUNTF if and only if $\lambda, N$ and $M$ satisfies (3.14).

We mention that Corollary 3.10 was subsequently and independently proved in [6].

Remark 5. (a) Let $N, M \in \mathbb{N}$, and let $D$ be the common divisors of $N$ and $M$. By Corollary 3.10 we see that $\operatorname{STF}(N, M)$ produces a FUNTF if and only if $M \geq 2 N-1$ or $M=2 N-d$ for some $d \in D$. This result is somewhat disappointing since it implies that spectral tetris frames with redundancy below two only are realizable by taking unions of spectral tetris frames for subspaces, where the number of vectors for each subspace frame is two times the subspace dimension minus one.

(b) Consider the frame $\operatorname{STF}(4,11)$ from (3.11). Since $\lambda=11 / 4, N=4$, and $M=11$ satisfies (3.14), it follows from Corollary 3.10 that $\operatorname{STF}(4, \tilde{M})$ with $\tilde{M}=M-N=7$ produces a FUNTF. Moreover, we see that $\operatorname{STF}(4,11)=\operatorname{STF}(4,7) \cup E_{4}$.

\section{Acknowledgments}

The authors would like to thank Jameson Cahill for valuable discussions. K. A. Okoudjou was supported by ONR grants: N000140910324 \& N000140910144, and by the Alexander von Humboldt foundation. He would also like to express his gratitude to the Institute for Mathematics at the University of Osnabrück for its hospitality while part of this work was completed. 


\section{References}

[1] B. Alexeev, J. Cahill, D. G. Mixon, Full spark frames, preprint.

[2] J. J. Benedetto, M. Fickus, Finite normalized tight frames, Adv. Comput. Math. 18 (2003), $357-385$.

[3] J. J. Benedetto, J. D. Kolesar, Geometric properties of Grassmannian frames for $\mathbb{R}^{2}$ and $\mathbb{R}^{3}$, EURASIP J. Applied Signal Processing, 2006, pp. 1-17.

[4] R. Calderbank, P. G. Casazza, A. Heinecke, G. Kutyniok, A. Pezeshki, Fusion frames: existence and construction, Adv. Comput. Math. 35 (2011), 1-31.

[5] P. G. Casazza, M. Fickus, D. G. Mixon, Y. Wang, and Z. Zhou, Constructing tight fusion frames, Appl. Comput. Harmon. Anal. 30 (2011), 175-187.

[6] P. Casazza, A. Heinecke, K. Kornelson, Y. Wang, and Z. Zhou, Necessary and sufficient conditions to perform Spectral Tetris, preprint.

[7] P. G. Casazza and J. Kovačević, Equal-norm tight frames with erasures, Adv. Comput. Math. 18 (2003), 387-430.

[8] T.-Y. Chien and S. Waldron, A classification of the harmonic frames up to unitary equivalence, Appl. Comput. Harmon. Anal. 30 (2011), 307-318.

[9] O. Christensen, "An Introduction to Frames and Riesz Bases," Applied and Numerical Harmonic Analysis. Birkhäuser Boston, Inc., Boston, MA, 2003.

[10] V. K. Goyal, J. Kovačević, and J. A. Kelner, Quantized frame expansions with erasures, Appl. Comput. Harmon. Anal. 10, 203-233 (2001).

[11] J. Kovačević and A. Chebira, Life Beyond Bases: The Advent of Frames (Part I), IEEE Signal Proces. Magazine 24 (2007), 86-104.

[12] J. Kovačević and A. Chebira, Life Beyond Bases: The Advent of Frames (Part II), IEEE Signal Proces. Magazine 24 (2007), 115-125.

[13] G. Kutyniok, F. Krahmer, and J. Lemvig, Sparsity and spectral properties of dual frames, preprint.

[14] T. Y. Lam and K. H. Leung, On vanishing sums of roots of unity, J. Algebra 224 (2000), 91-109.

[15] G. Sivek, On vanishing sums of distinct roots of unity, Integers 10 (2010), 365-368.

[16] N. Strawn, Finite frame varieties: Nonsingular points, tangent spaces, and explicit local parametrization, J. Fourier Anal. Appl. 17 (2011), 821-853.

[17] T. Strohmer and R. W. Heath, Grassmannian frames with applications to coding and communication, Appl. Comput. Harmon. Anal. 14 (2003), 257-275. 
[18] L. R. Welch, Lower bounds on the maximum cross correlation of signals, IEEE Trans. Inform. Theory 20 (1974), 397-399.

[19] P. Xia, S. Zhou, and G. B. Giannakis, Achieving the Welch bound with difference sets, IEEE Trans. Inform. Theory 51 (2005), no. 5, 1900-1907. 